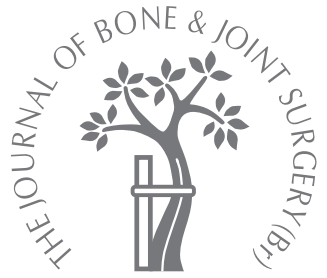

R. Madhu,

R. Kotnis,

A. Al-Mousawi,

N. Barlow,

S. Deo,

P. Worlock,

K. Willett

From the Trauma

Service, John

Radcliffe Hospital,

Oxford, England

n. Madhu, MRCS, Research

Fellow in Trauma

R. Kotnis, MRCS, Specialist

Registrar in Trauma

A. Al-Mousawi, MBBS,

Senior House Officer in Trauma

N. Barlow, MBBS, Senior

House Officer in Trauma

K. Willett, FRCS, Professor of

Orthopaedic Trauma Surgery

John Radcliffe Hospital,

Headington, Oxford OX3 9DU,

UK.

In. Deo, FRCS(Trauma \&

Orth), Consultant in Trauma

and Orthopaedics

The Great Western Hospital,

Marlborough Road, Swindon,

Wiltshire SN3 6BB, UK.

- P. Worlock, DM, FRCS,

Consultant in Trauma and

Orthopaedics

Department of Orthopaedic

Surgery, Newcastle Genera

Hospital, Westgate Road,

Newcastle upon Tyne, NE4 6BE,

UK.

Correspondence should be sent to Professor K. Willett; e-mail:

Keith.willett@ndos.ox.ac.uk

(C)2006 British Editorial Society of Bone and Joint Surgery doi:10.1302/0301-620X.88B9.

$17588 \$ 2.00$

$J$ Bone Joint Surg [Br]

2006;88-B:1197-203.

Received 29 December 2005;

Accepted after revision 18 May

2006

\title{
Outcome of surgery for reconstruction of fractures of the acetabulum
}

\author{
THE TIME DEPENDENT EFFECT OF DELAY
}

This is a retrospective case review of 237 patients with displaced fractures of the acetabulum presenting over a ten-year period, with a minimum follow-up of two years, who were studied to test the hypothesis that the time to surgery was predictive of radiological and functional outcome and varied with the pattern of fracture. Patients were divided into two groups based on the fracture pattern: elementary or associated. The time to surgery was analysed as both a continuous and a categorical variable. The primary outcome measures were the quality of reduction and functional outcome. Logistic regression analysis was used to test our hypothesis, while controlling for potential confounding variables.

For elementary fractures, an increase in the time to surgery of one day reduced the odds of an excellent/good functional result by $15 \%(p=0.001)$ and of an anatomical reduction by $18 \%$ ( $p=0.0001$ ). For associated fractures, the odds of obtaining an excellent/good result were reduced by $19 \%(p=0.0001)$ and an anatomical reduction by $18 \%(p=0.0001)$ per day.

When time was measured as a categorical variable, an anatomical reduction was more likely if surgery was performed within 15 days (elementary) and five days (associated). An excellent/good functional outcome was more likely when surgery was performed within 15 days (elementary) and ten days (associated).

The time to surgery is a significant predictor of radiological and functional outcome for both elementary and associated displaced fractures of the acetabulum. The organisation of regional trauma services must be capable of satisfying these time-dependent requirements to achieve optimal patient outcomes.

The incidence of acetabular and pelvic fracture is about 1 in 50000 of the population per year in the United Kingdom ${ }^{1}$ and constitutes approximately $2 \%$ to $3 \%$ of all fractures. ${ }^{2,3}$ Operative management of displaced fractures has become the standard treatment in centres providing a pelvic and acetabular service.

The outcome from fracture of the acetabulum may be influenced by many factors. The surgeon is unable to control age, fracture pattern, the presence of critical chondral damage or damage to the femoral head, dislocation of the hip at the time of injury, damage to the sciatic nerve and patient comorbidities. However, the timing of surgery, the surgical approach, the quality of reduction and the avoidance of loss of reduction ${ }^{4}$ are within the influence of the surgeon.

Failure to achieve anatomical reduction and rigid internal fixation leads to a poorer functional outcome and an increase in posttraumatic arthritis. ${ }^{5}$ Letournel and Judet ${ }^{6}$ identified a residual step in the articular surface of more than $2 \mathrm{~mm}$ as a significant factor in determining outcome. It has been stated that anatomical reduction is more difficult to achieve as the timing of surgery increases. ${ }^{7,8}$ Mears et $\mathrm{al}^{7}$ found that after 11 days, surgery led to significantly fewer anatomical reductions.

In this study, we tested the hypothesis that the time to surgery is a predictor of radiological and functional outcome for both elementary and associated patterns of fracture. It was undertaken at an institution which is not formally funded to provide a regional trauma service and is unable to prioritise tertiary referrals. Such cases compete with local patients for access, often creating non-clinical delays in the transfer for operation during the study period. Previous studies have reported on the effects of a delay in surgery for displaced fractures of the acetabulum as a group. ${ }^{1,4,5,7}$

\section{Patients and Methods}

This retrospective case review was conducted on all patients who underwent internal fixation 
Table I. The distribution of fracture types and the surgical approach used (number of patients and percentage)

\begin{tabular}{|c|c|c|c|}
\hline Elementary fracture types & & Associated fracture types & \\
\hline Anterior column & $17(6.6)$ & Posterior wall + posterior column & $10(3.9)$ \\
\hline Posterior wall & $44(17.3)$ & Anterior + posterior column & $64(25)$ \\
\hline Posterior column & 9 (3.5) & Anterior column + posterior hemitransverse & $20(7.8)$ \\
\hline Transverse & $35(13.7)$ & Transverse and posterior wall & $45(17.7)$ \\
\hline Anterior wall & 0 & T-shaped & $10(3.9)$ \\
\hline \multicolumn{4}{|l|}{ Surgical approach used } \\
\hline Kocher-Langenbeck (KL) & 123 patients $(48)$ & & \\
\hline Ilioinguinal (IL) & 54 patients $(21)$ & & \\
\hline Triradiate & 32 patients (14) & & \\
\hline Combined $\mathrm{KL}$ and IL & 39 patients $(15)$ & & \\
\hline Extended iliofemoral & 6 patients (2) & & \\
\hline
\end{tabular}

of displaced fractures of the acetabulum between March 1991 and July 2003 in the trauma unit of a university teaching hospital, for whom there was a minimum followup of two years. We excluded patients who underwent fixation elsewhere prior to transfer.

There were 254 consecutive patients with a mean age was 36.7 years ( 17 to 81 ) and there were 212 men and 42 women. The mechanism of injury was a road traffic accident in 193 patients $(76 \%)$ and a fall in 61 (24\%). The pattern of injury was an isolated acetabular injury in 61 patients (24\%), acetabular and limb fracture in 98 $(38.5 \%)$, an associated cerebral or visceral injury in 42 $(16.5 \%)$ and polytrauma in $53(21 \%)$. A dislocation of the hip joint at the time of injury was recorded in 108 patients $(42.5 \%)$. All had a closed reduction of the dislocation within six hours, except for one patient who was referred with an irreducible dislocation.

Our institution functions as a tertiary referral centre for acetabular and pelvic injuries: 190 patients (74.8\%) were transferred to our unit from other hospitals. Of these, 62 $(24.4 \%)$ were from outside our designated geographical catchment area.

Internal fixation was performed if there was $2 \mathrm{~mm}$ or more of displacement, if intra-articular fragments were identified, and when instability or incongruence of the hip joint or an irreducible fracture dislocation were found.

Radiological investigations prior to definitive surgery included an anteroposterior radiograph, Judet $45^{\circ}$ oblique views of the pelvis ${ }^{9}$ and a CT scan with $2 \mathrm{~mm}$ cuts to visualise the whole pelvis. From these investigations, the fracture patterns were classified according to the criteria of Letournel and Judet. ${ }^{10}$ Of the 254 fractures, 105 (41\%) were elementary and 149 (59\%) were associated fracture types. The distribution is shown in Table I.

Internal fixation was performed by one of three surgeons (KW, PW, RK) with a special interest in pelvic and acetabular reconstruction. The goal was to achieve an anatomical reduction of the innominate bone and the articular surface of the acetabulum as early as possible following the injury. The surgical approaches used are summarised in Table I. Extensile or combined approaches were used in 77 patients $(30 \%)$ where indirect reduction of the fracture was consid- ered unachievable through a simple ilioinguinal or KocherLangenbeck approach because of early callus formation. In cases where joint salvage was deemed impossible, restoration of the columns for subsequent total hip replacement (THR) was the aim. Reduction and fixation were achieved using a combination of lag screws and reconstruction plates. Cerclage wires were occasionally used.

The articular surface of the head of the femur was noted to be damaged in 38 patients $(15 \%)$, intra-articular loose fragments were found in $22(9 \%)$, major contusion of the sciatic nerve had occurred in $28(11 \%)$, and the acetabular articular cartilage had been impacted in $25(10 \%)$.

Patients were given oral indomethacin, $25 \mathrm{mg}$ three times a day for three weeks, unless there were specific contraindications (eg. gastrointestinal haemorrhage or dyspepsia). Weight-bearing after operation was dependent on fracture comminution, the stability of the fixation, and satisfactory radiographs. Most patients were allowed touch weightbearing on the affected side during the initial six weeks, partial weight-bearing to a maximum of $15 \mathrm{~kg}$ from six weeks onward and full weight-bearing by 12 weeks.

Post-operative complications were divided into local and systemic. Local complications included superficial wound infection, deep wound infection requiring re-exploration, neurological impairment, heterotopic ossification and loss of reduction. Systemic complications included bronchopneumonia, thromboembolism, systemic sepsis and acute respiratory distress syndrome.

All patients were followed up clinically and radiologically by one of the three operating surgeons in a dedicated clinic at six and 12 weeks, and six and 12 months with a further review at two years following operation. The mean period of follow-up was 2.9 years ( 2 to 4 ). The presence or absence of heterotopic ossification, degenerative changes and avascular necrosis in the injured hip were recorded. The two-year follow-up was the point used for analysis of outcome.

The quality of reduction based on plain radiographs was assessed at each follow-up using our own system. Anatomical reduction was defined as having no step in the joint surface or a gap of less than $2 \mathrm{~mm}$. Adequate reduction was defined as a step of $2 \mathrm{~mm}$ or less or a gap of $2 \mathrm{~mm}$. Poor 
Table II. The number of patients operated on in the time intervals for both patterns of fracture

\begin{tabular}{llllclc}
\hline Time period (days) & $\mathbf{0}$ to $\mathbf{5}$ & $\mathbf{6}$ to $\mathbf{1 0}$ & $\mathbf{1 1}$ to $\mathbf{1 5}$ & $\mathbf{1 6}$ to $\mathbf{2 0}$ & $\mathbf{>} \mathbf{2 0}$ & Total \\
\hline Elementary & 24 & 32 & 20 & 9 & 6 & 91 \\
Associated & 44 & 47 & 35 & 15 & 5 & 146
\end{tabular}

reduction described a step or gap of more than $2 \mathrm{~mm}$. For statistical analysis, the three categories were dichotomised into two categories, anatomical or adequate and poor.

The functional outcome was scored using the system described by Merle D'Aubigne and Postel. ${ }^{11}$ For statistical analysis these categories were also dichotomised into excellent and good, or moderate and poor as described by Merle D’Aubigné and Postel. ${ }^{11}$

The interval in days between the date of injury and definitive fixation was defined as the time to surgery and measured as both a continuous and a categorical variable ( 1 to 5,6 to 10,11 to 15,16 to $20,>20$ days), as this was anticipated to be a non-linear relationship. The quality of reduction and functional outcome for both types of fracture were recorded with respect to the time of surgery.

Statistical analysis was performed independently using the Stata program, version 9.1 (Stata Corporation, College Station, Texas), with logistic regression to control the potential confounding variables of age, the presence of hip dislocation, sciatic nerve injury and associated injuries. A $\mathrm{p}$ value $<0.05$ was considered to be significant.

\section{Results}

Of the 254 patients, 17 had incomplete data, leaving the results of 237 patients for analysis. The number of patients operated on in each time period is shown in Table II.

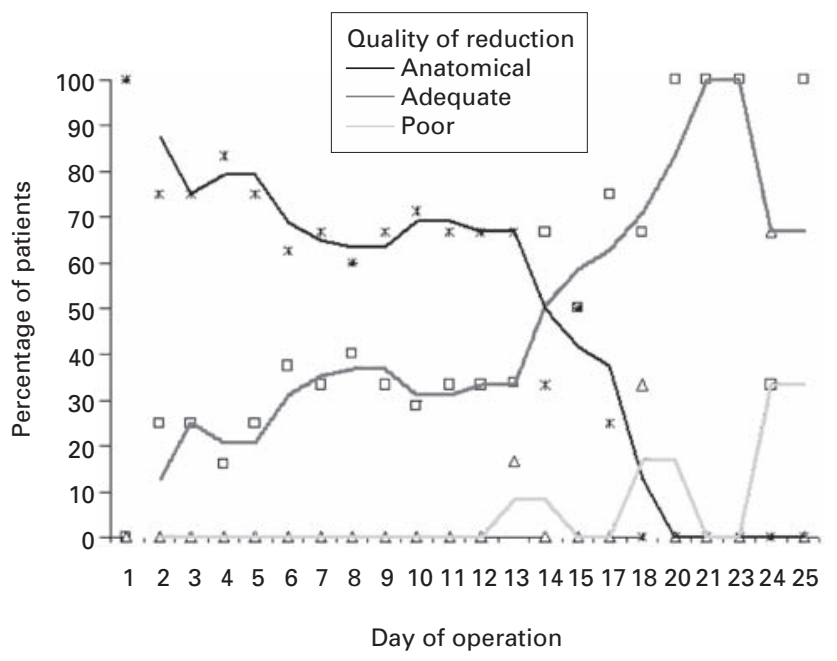

Fig. 1

The relationship between the timing of surgery and the quality of reduction for elementary fracture types.
Combined results for all types of acetabular fracture

Radiological. For both fracture types combined, review at a mean of 2.9 years (2 to 4 ) demonstrated that $48.9 \%$ of patients (116) achieved an anatomical, 41.3\% (98) an adequate and $9.7 \%(23)$ a poor reduction.

Functional. Overall, $76 \%$ of patients (180) achieved excellent/good results and 24\% (57) moderate/poor.

Analysing time to surgery as a continuous variable

Radiological outcome. The quality of reduction with respect to time to surgery is demonstrated in Figure 1 for elementary fractures and Figure 2 for associated fractures. Statistical analysis (Table III) showed that the average daily reduction of the odds of attaining an anatomical reduction was $18 \%$ (odds ratio $(\mathrm{OR})=0.82$ ) for both fracture patterns. However, Figures 1 and 2 demonstrate that this relationship is non-linear (see analysis of time to surgery as a categorical variable). Adjusting for age, dislocation, injury to the sciatic nerve and associated injuries made minimal difference to the results for both patterns of fracture.

Functional outcome. Figures 3 and 4 show the functional outcome for each pattern of fracture with respect to time to surgery. The average daily reduction of the odds of attaining an excellent/good outcome was $15 \%(\mathrm{OR}=0.85)$ for elementary fractures and $19 \%(\mathrm{OR}=0.81)$ for associated types (Table III). However, Figures 3 and 4 also demonstrate that this relationship is non-linear. Adjusting for age, dislocation, injury to the sciatic nerve and associated injuries made minimal difference to the results for both patterns of fracture.

\section{Analysing time to surgery as a categorical variable}

Radiological outcome. Table IV shows the results for both types of fracture in each time period.

For elementary fractures, there was no significant difference in the odds of obtaining an anatomical reduction when

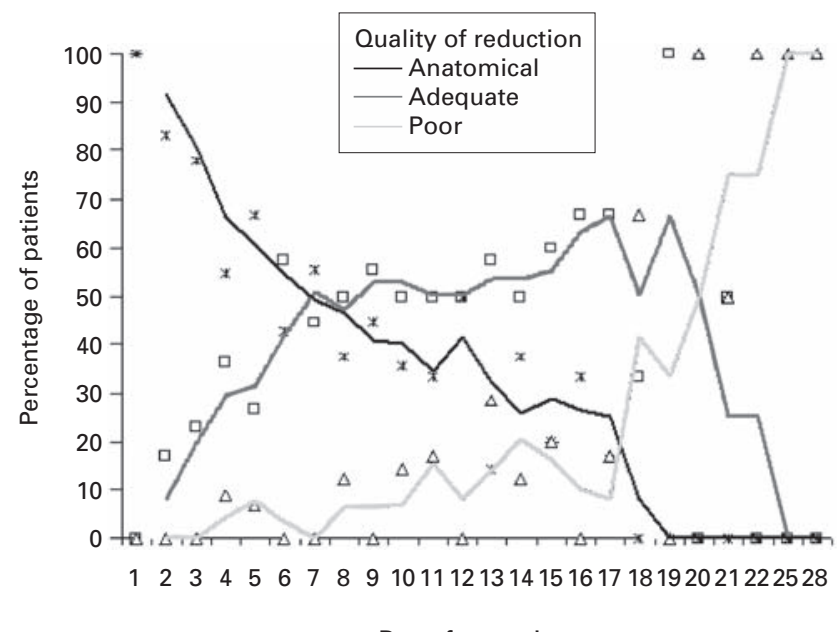

Day of operation

Fig. 2

The relationship between the timing of surgery and the quality of reduction for associated fracture types. 
Table III. The relationship of anatomical reduction and excellent/good functional outcome with time to surgery measured as a continuous variable. Adjusted results were values obtained when controlling for confounding variables (age, hip dislocation, sciatic nerve injury, associated injuries)

\begin{tabular}{|c|c|c|c|}
\hline & Odds ratios & $\begin{array}{l}95 \% \text { Confidence } \\
\text { interval }\end{array}$ & $p$ value \\
\hline \multicolumn{4}{|l|}{ Elementary fractures } \\
\hline Radiological-anatomical reduction & 0.82 & 0.74 to 0.90 & $<0.0001$ \\
\hline Radiological-adjusted results & 0.81 & 0.74 to 0.90 & $<0.0001$ \\
\hline Function-excellent/good & 0.85 & 0.77 to 0.94 & 0.001 \\
\hline Function-adjusted results & 0.85 & 0.77 to 0.95 & 0.004 \\
\hline \multicolumn{4}{|l|}{ Associated fractures } \\
\hline Radiological-anatomical reduction & 0.82 & 0.76 to 0.89 & $<0.0001$ \\
\hline Radiological-adjusted results & 0.82 & 0.75 to 0.89 & $<0.0001$ \\
\hline Function-excellent/good & 0.81 & 0.75 to 0.88 & $<0.0001$ \\
\hline Function-adjusted results & 0.79 & 0.72 to 0.87 & $<0.0001$ \\
\hline
\end{tabular}

comparing patients operated on in the time periods 1 to 5,6 to 10 and 11 to 15 days. Significance was first reached when the time to surgery was more than 15 days $(p=0.04)$. For associated fractures, significance was first reached when the time to surgery was more than five days $(\mathrm{p}=0.008)$.

Functional outcome. Table $\mathrm{V}$ shows the results for both types of fracture in each time period.

For elementary fractures, there was no significant difference in the odds of obtaining an anatomical reduction when comparing patients operated on in the periods 1 to 5,6 to 10 and 11 to 15 days. Significance was first reached when the time to surgery was more than 15 days $(p=0.02)$. For associated fractures, significance was first reached when the time to surgery exceeded ten days $(\mathrm{p}<0.0001)$.

Complications. The local and systemic complications for both types of fracture are shown in Table VI. Only deep infection and heterotopic ossification demonstrated a time-

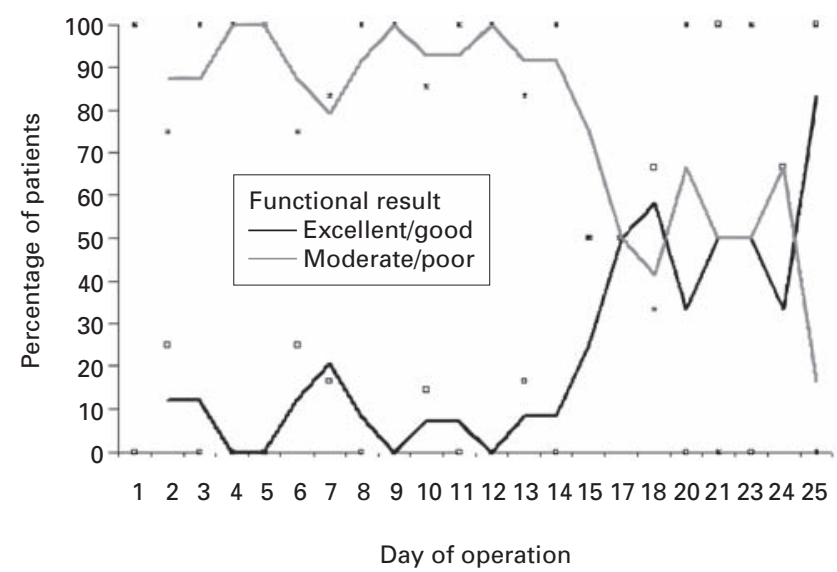

Fig. 3

The relationship between the timing of surgery and the functional outcome for elementary fracture types. dependent relationship, but the results did not reach significance. The odds of deep wound infection increased by $10 \%$ per day for elementary fractures $(\mathrm{OR}=1.1,95 \%$ Confidence interval (CI) 0.92 to $1.31, p=0.3$ ) and $20 \%$ for associated fractures $(\mathrm{OR}=1.2,95 \%$ CI 1.0 to $1.33, \mathrm{p}=$ $0.04)$. The odds of heterotopic ossification increased by $10 \%$ for elementary fractures $(\mathrm{OR}=1.1,95 \%$ CI 0.95 to $1.18, \mathrm{p}=0.3)$ and $10 \%$ for associated fractures $(\mathrm{OR}=1.1$, $95 \%$ CI 0.97 to $1.15, \mathrm{p}=0.2$ ).

\section{Discussion}

The aim of treatment in displaced fractures of the acetabulum is to obtain an accurate and stable anatomical reduction and a functional, mobile and painless hip joint. ${ }^{5}$ It has been well documented that the accuracy of reduction correlates with outcome and with a reduced risk of post-traumatic osteoarthritis of the hip. ${ }^{7}$

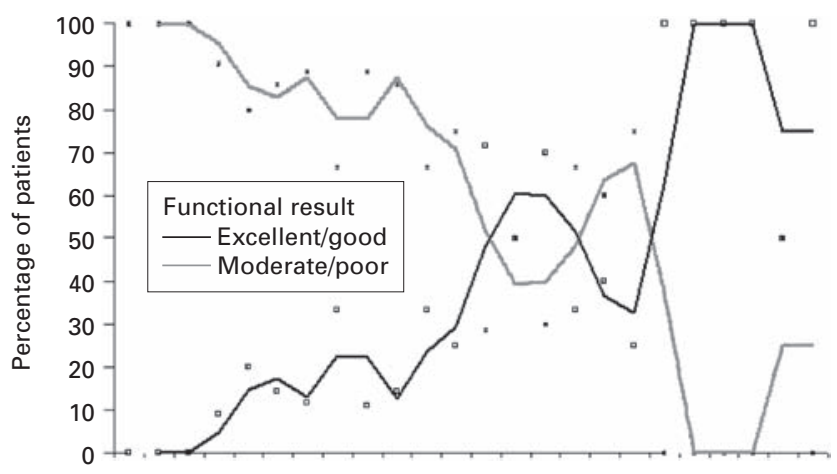

1223445667889101112131415161718192021222528

Day of operation

Fig. 4

The relationship between the timing of surgery and the functional outcome for associated fracture types. 
Table IV. The quality of reduction when measuring time to surgery as a categorical variable (days). The results for the time period one to five days have been compared with the other time periods

\begin{tabular}{|c|c|c|c|c|c|}
\hline \multirow{2}{*}{$\begin{array}{l}\text { Time period } \\
\text { (days) }\end{array}$} & \multicolumn{2}{|l|}{ Radiological } & \multirow[b]{2}{*}{ Odds ratio } & \multirow{2}{*}{$\begin{array}{l}95 \% \text { Confidence } \\
\text { Interval }\end{array}$} & \multirow[b]{2}{*}{$p$ value } \\
\hline & Anatomical & Adequate/poor & & & \\
\hline & Elementary fractures & Elementary fractures & & & \\
\hline 1 to 5 & 19 & 5 & - & - & - \\
\hline 6 to 10 & 21 & 11 & 0.50 & 0.15 to 1.7 & 0.3 \\
\hline 11 to 15 & 11 & 9 & 0.32 & 0.09 to 1.2 & 0.09 \\
\hline 16 to 20 & 1 & 8 & 0.03 & 0.003 to 0.3 & 0.004 \\
\hline \multirow[t]{2}{*}{$>20$} & 0 & 6 & - & - & - \\
\hline & Associated fractures & Associated fractures & & & \\
\hline 1 to 5 & 31 & 13 & - & - & - \\
\hline 6 to 10 & 20 & 27 & 0.31 & 0.13 to 0.74 & 0.008 \\
\hline 11 to 15 & 10 & 25 & 0.17 & 0.06 to 0.44 & $<0.0001$ \\
\hline 16 to 20 & 2 & 13 & 0.06 & 0.01 to 0.33 & 0.001 \\
\hline$>20$ & 0 & 5 & - & - & - \\
\hline
\end{tabular}

Table V. The functional outcome when measuring time to surgery as a categorical variable (days). The results for the time period one to five days have been compared with the other time periods

\begin{tabular}{|c|c|c|c|c|c|}
\hline \multirow{2}{*}{$\begin{array}{l}\text { Time period } \\
\text { (days) }\end{array}$} & \multicolumn{2}{|l|}{ Function } & \multirow[b]{2}{*}{ Odds ratio } & \multirow{2}{*}{$\begin{array}{l}95 \% \text { Confidence } \\
\text { Interval }\end{array}$} & \multirow[b]{2}{*}{ p value } \\
\hline & Excellent/good $(n=180)$ & Moderate/poor $(n=57)$ & & & \\
\hline & Elementary fractures & Elementary fractures & & & \\
\hline 1 to 5 & 30 & 1 & - & - & - \\
\hline 6 to 10 & 28 & 4 & 0.3 & 0.03 to 2.9 & 0.30 \\
\hline 11 to 15 & 17 & 2 & 0.39 & 0.03 to 4.7 & 0.46 \\
\hline 16 to 20 & 1 & 4 & 0.05 & 0.005 to 0.6 & 0.02 \\
\hline \multirow[t]{2}{*}{$>20$} & 0 & 4 & 0.02 & 0.002 to 0.30 & 0.004 \\
\hline & Associated fractures & Associated fractures & & & \\
\hline 1 to 5 & 40 & 4 & - & - & - \\
\hline 6 to 10 & 39 & 8 & 0.49 & 0.14 to 1.75 & 0.27 \\
\hline 11 to 15 & 16 & 19 & 0.08 & 0.02 to 0.29 & $<0.0001$ \\
\hline 16 to 20 & 8 & 7 & 0.11 & 0.03 to 0.48 & 0.003 \\
\hline$>20$ & 1 & 4 & 0.03 & 0.002 to 0.28 & 0.003 \\
\hline
\end{tabular}

Delay in reduction and definitive fixation of these fractures results in an increase in the formation of scar tissue between bony fragments and the formation of early callus at the fracture site. In these circumstances the surgeon will be faced with a more difficult exposure of less mobile fracture fragments which are more difficult to reduce. ${ }^{2}$ Techniques of indirect reduction are less likely to be effective. The reduced likelihood of obtaining an accurate and stable anatomical reduction will lead to a poor outcome. Delay in fixation may also be detrimental to the viability of the femoral head in cases of persistent subluxation. ${ }^{6}$ There is an increase in the incidence of both chondrolysis and osteonecrosis of the head with delayed reconstruction. ${ }^{6}$

The timing of surgery in relation to displaced fractures of the acetabulum has been reported in the literature. Johnson et $\mathrm{al}^{12}$ described a rate of anatomical reduction of only $52 \%$ in fractures which were operated on more than 21 days from the time of injury, and recently Griffin, Beaule and Matta ${ }^{13}$ found that for associated fracture types the accuracy of reduction and the clinical results correlated significantly but the time to surgery did not.

Various authors have categorised the time to surgery to assess the relationship of a delay to surgery and outcome.
Table VI. Complications following surgery for both fracture patterns combined

\begin{tabular}{lrc}
\hline Complication & Number of patients & Percentage \\
\hline Local & & \\
$\quad$ Superficial wound infection & 19 & 8.0 \\
Wound infection ${ }^{*}$ (re-exploration) & 9 & 3.8 \\
$\quad$ Increased neurology post-surgery & 6 & 2.5 \\
Systemic & & \\
$\quad$ Acute respiratory distress syndrome & 8 & 3.3 \\
$\quad$ Pneumonia & 9 & 3.8 \\
$\quad$ Thromboembolic & 12 & 5.1 \\
$\quad$ Sepsis & 5 & 2.2 \\
Simple fracture types (n = 91) & & \\
$\quad$ Heterotopic ossification & 9 & 9.8 \\
$\quad$ Avascular necrosis & 5 & 5.4 \\
$\quad$ Osteoarthritis & 11 & 12.1 \\
Complex fracture types (n = 146) & & \\
$\quad$ Heterotopic ossification & 19 & 13 \\
$\quad$ Avascular necrosis & 8 & 5.5 \\
$\quad$ Osteoarthritis & 21 & 14.4 \\
Heterotopic ossification & & \\
$\quad$ Overall heterotopic ossification rate & 28 & 11.8 \\
$\quad$ Overall avascular necrosis rate & 13 & 13.5 \\
$\quad$ Overall osteoarthritis rate & 32 &
\end{tabular}

* only wound infection and heterotopic ossification had a time-dependent relationship 
Mears et $\mathrm{al}^{7}$ categorised time periods into less than two days from injury, 3 to 10 days and 11 to 21 days. They reported that patients with a delay of more than 11 days had a significantly lower rate of anatomical reduction. Matta $^{5}$ divided the time to surgery into three periods: 1 to 7 days, 8 to 14 days and 15 to 21 days. Of the 259 patients in his study, there was a significant difference in the quality of the reduction obtained when comparing patients operated on days 1 to 14 with those undergoing fixation at between 15 and 21 days.

Previous studies have described the effect of a delay in surgery for displaced fractures as a whole group and only measured time as a categorical variable. In this study we assessed the effect of the time to surgery for both elementary and associated types individually, and the category 'time' was treated as both a continuous and a categorical variable. The latter was performed to account for the nonlinear relationship between time to surgery and the logodds of outcome.

Our results when analysing time to surgery as a continuous variable show that for both patterns of fracture the odds of obtaining an anatomical reduction and an excellent/good functional outcome reduce significantly as the time to surgery increases (Table III). Figures 1 and 2 show that an anatomical reduction was no longer probable after day 14 for elemental fractures and day six for associated fractures. Figures 3 and 4 show that an excellent/good functional outcome was most likely in those patients operated on within 15 days for elementary fractures and ten days for associated fractures. The results when analysing time as a categorical variable (Tables IV and V) show a similar relationship between time to surgery and outcome for both patterns of fracture.

Matta $^{5}$ also drew attention to other factors which influence the outcome of patients with displaced fractures of the acetabulum such as injuries of the femoral head, increasing patient age and operative complications. Murphy et $\mathrm{al}^{14}$ studied prognostic factors and their relationship to the functional outcome of fractures of the acetabulum. He identified four dominant prognostic factors which were associated with a suboptimal outcome: complex (associated) fracture type, imperfect reduction, the presence of local complications, and heterotopic bone. Factors which were not significant were gender, palsy of the sciatic nerve and dislocation of the hip. The clinical results in patients with associated injuries have been reported to be similar to those with an isolated fracture. ${ }^{5,15}$ In our study, age, dislocation of the hip, injury to the sciatic nerve and associated injuries had no significant effect on the odds ratio or $95 \%$ CI. Only deep infection requiring re-exploration and heterotopic bone demonstrated time-dependent trends, but these were not statistically significant.

The strengths of our study are the large number of patients in a single institution, the high follow-up $(93.3 \%)$ at a mean of 2.9 years ( 2 to 4 ); review of the patients to an agreed protocol, control of potential confounding varia- bles, the categorisation of time periods, the frequency of delay to surgery and the analysis of results for both elementary and associated types of fracture. The limitations include the retrospective nature, non-blinded assessors at follow-up, the potential inter-observer variability when assessing radiological outcome using plain radiographs (as opposed to CT), and the smaller numbers of patients who underwent surgery in the later time periods. Although longer follow-up may yield more reliable data on the rates of hip replacement, the policy of our department is to discharge patients who have no significant complications or complaints after two years. This is based on the predictive value of the two-year assessment as described by Letournel and Judet ${ }^{6}$ and Letournel. ${ }^{10}$

It is worth noting that 190 of the 254 patients $(74.8 \%)$ were referred to our institution from other hospitals and constituted the majority of those operated on after two weeks. The main cause of delay was the lack of availability of beds to permit unrestricted transfer to our hospital. Delay in initiating referral was also a contributing factor. Bircher and Giannoudis ${ }^{16}$ highlighted the shortcomings of the pelvic and acetabular trauma service in the United Kingdom.

This study describes the effect of delay to surgery on outcome. The odds of achieving an anatomical reduction and an excellent/good functional outcome reduces significantly for both elementary and associated displaced fractures of the acetabulum as the time to surgery increases. Delays in definitive fixation have more effect on outcome for the associated fracture subgroup. For management to be effective, definitive surgery should be performed as soon as possible. Regional and national trauma referral systems must be capable of achieving time dependent targets to optimise patient outcome.

The authors wish to thank Richard Keys, Consultant Trauma Surgeon, John Radcliffe Hospital for allowing his patients to be included in this study, Nicola Alder from the Centre for Statistics in Medicine, Wolfson College, University of Oxford, for help with the statistical sections of the paper, and Usha Chouhan from the Medical Records department, John Radcliffe Hospital, for her help with locating patient records.

No benefits in any form have been received or will be received from a commercial party related directly or indirectly to the subject of this article.

\section{References}

1. Laird A, Keating JF. Acetabular fractures: a 16-year prospective epidemiological study. J Bone Joint Surg [Br] 2005;87-B:969-73.

2. Mucha P Jr, Farnell MB. Analysis of pelvic fracture management. J Trauma 1984; 24:379-86.

3. Pohlemann T, Tscherne H, Baumgartel F, et al. Pelvic fractures: epidemiology, therapy and long-term outcome: overview of the multicentre study of the Pelvis Study Group. Unfallchirurg 1996;99:160-7.

4. Giannoudis PV, Grotz MR, Papakostidis C, Dinopoulos H. Operative treatment of displaced fractures of the acetabulum: a meta-analysis. J Bone Joint Surg $[\mathrm{Br}]$ 2005:87-B:2-9.

5. Matta JM. Fractures of the acetabulum: accuracy of reduction and clinical results in patients managed operatively within three weeks after the injury. J Bone Joint Surg [Am] 1996;78-A:1632-45.

6. Letournel E, Judet R. Fractures of the acetabulum. Second ed. Berlin: Springer Verlag, 1993:29-62.

7. Mears DC, Velyvis JH, Chang CP. Displaced acetabular fractures managed operatively: indicators of outcome. Clin Orthop 2003;407:173-86.

8. Deo SD, Tavares SP, Pandey RK, et al. Operative management of acetabular fractures in Oxford. Injury 2001;32:581-6. 
9. Judet R, Judet J, Letournel E. Fractures of the acetabulum: classification and surgical approaches for open reduction: preliminary report. J Bone Joint Surg [Am]1964; 46-A:1615-46.

10. Letournel E. Acetabulum fractures: classification and management. Clin Orthop 1980;151:81-106.

11. Merle d'Aubigné R, Postel M. Functional results of hip arthroplasty with acrylic prosthesis. J Bone Joint Surg [Am] 1954;36-A:451-75.

12. Johnson EE, Matta JM, Mast JW, Letournel E. Delayed reconstruction of acetabular fractures 21 to 120 days following injury. Clin Orthop 1994;305:20-30.
13. Griffin DB, Beaule PE, Matta JM. Safety and efficacy of the extended iliofemoral approach in the treatment of complex fractures of the acetabulum. J Bone Joint Surg [Br] 2005;87-B:1391-6.

14. Murphy D, Kaliszer M, Rice J, McElwain JP. Outcome after acetebular fractures: prognostic factors and their inter-relationships. Injury 2003;34:512-17.

15. Liebergall M, Mosheiff R, Low J, et al. Acetabular fractures: clinical outcome of surgical treatment. Clin Orthop 1999;366:205-16.

16. Bircher M, Giannoudis PV. Pelvic trauma management within the UK: a reflection of a failing trauma service. Injury 2004;35:2-6. 